\title{
On the Maximization of the Fundamental Eigenvalue in Topology Optimization
}

\author{
Wolfgang Achtziger* Michal Kočvara**
}

April 26, 2006

\begin{abstract}
The paper considers a classic formulation of the topology optimization problem of discrete or discretized structures. The objective function to be maximized is the smallest natural frequency of the structure. The model of the problem takes into account multiple load conditions, equilibrium of forces, constraints on compliance and volume, and the effect of possible non-structural mass. After the statement of the mathematical model we discuss serious obstacles for a successful numerical treatment of this formulation such as non-Lipschitzean behavior and even discontinuity of the objective function. As a cure we present an equivalent reformulation as a bilinear semidefinite programming problem without the pitfalls of the original problem. An algorithm is presented for finding an approximation of a globally optimal solution up to a user-defined accuracy. The key ingredient of this algorithm is the treatment of a sequence of linear SDPs. Numerical examples are provided for truss structures. Examples of both academic and larger size illustrate the theoretical results achieved and demonstrate the practical use of this approach. We conclude with an extension on multiple non-structural mass conditions.
\end{abstract}

Keywords: Topology optimization, Vibration of structures, Optimization of Eigenvalues, Nonlinear semidefinite programming

Mathematics Subject Classification (2000): 74P05, 74H45, 90C22

\section{Introduction}

The subject of this paper is topology optimization of discrete and discretized structures with consideration of free vibrations of the optimal structure. Maximization of the fundamental

*Department of Mathematics, Technical University of Denmark, Matematiktorvet, Building 303, $2800 \mathrm{Kgs}$. Lyngby, Denmark, on leave from Institute of Applied Mathematics, University of Dortmund, Vogelpothsweg 87, 44221 Dortmund, Germany, Email: wolfgang.achtziger@uni-dortmund.de

** Institute of Information Theory and Automation, Academy of Sciences of the Czech Republic, Pod vodárenskou věží 4, 18208 Prague 8 and Czech Technical University, Faculty of Electrical Engineering, Technická 2, 16627 Prague 6, Czech Republic, Email: kocvara@utia.cas.cz 
eigenvalue of a structure is a classic problem of structural engineering. The (generalized) eigenvalue problem typically reads as

$$
K(x) w=\lambda\left(M(x)+M_{0}\right) w
$$

where $K(x)$ and $M(x)$ are symmetric and positive semidefinite matrices that continuously (often linearly) depend on the parameter $x$. The main difficulty brings the nonsmooth dependence of eigenvalues on this parameter. In fact, we shall see below that the dependence of the smallest eigenvalue on $x$ may even be discontinuous in topology optimization problems.

The problem has been treated in the engineering literature since the beginning of 70s; see the paper [18] and the overview [17] summarizing the early development. See also the recent book [19] for up-to-date bibliography on this subject. The general problem of eigenvalue optimization belongs also to classic problems of linear algebra. When the matrix $M(x)+M_{0}$ is positive definite for all $x$, then one can resort to the theory developed for the standard eigenvalue problem; see [13] for an excellent overview. Not many papers studying the dependence of the eigenvalues on a parameter are available for the general case when $M(x)+M_{0}$ is only positive semidefinite; see, e.g. [6, 20, 22].

The paper is organized as follows. In Section 2 we present a formulation of the structural design problem where we maximize the fundamental frequency, i.e., the smallest eigenvalue of certain generalized eigenvalue problem, subject to equilibrium conditions and constraints on the volume and the compliance. We illustrate several severe theoretical difficulties of this formulation as non-Lipschitzean behavior and even discontinuity of the involved functions. In Section 3 we formulate this problem as a semidefinite program (SDP) with a bilinear matrix inequality (BMI) constraint. This formulation, however straightforward, has never been used for the numerical solution of the problem, up to our knowledge. The reason for this was the lack of available SDP-BMI solvers. We solve the problem by a recently developed code PENBMI [10]. Due to the BMI, the reformulated problem is nonconvex. By consideration of a related convex SDP, however, it is possible to improve lower and upper bounds for the globally optimal function value of the original problem. This, finally, leads to an algorithm for finding an approximation of a globally optimal solution of the original problem up to a given acurracy. Section 5 presents some numerical examples of different size. These examples illustrate the formulations and theoretical results developed in the paper and also demonstrate the solvability of the SDP formulations and thus their practical usefulness. In Section 6 the paper closes with an extension to problems with several independent non-structural masses applied at different time points.

All formulations and theorems in the presentation are developed for problems using discrete or discretized structural models satisfying certain properties. All numerical examples show trusses to keep the notation and visualization fixed and simple. The theory, however, also applies to discretized structures, for instance, to the variable thickness sheet or the free material optimization problems (see, e.g., [4]).

This paper is based on a mathematically oriented paper of the authors (see [2]). Here we want to present material and new examples relevant for practicioners.

We use standard notation. In particular, the $k \times k$ identity matrix is denoted by $I_{k \times k}$, and $\operatorname{ker}(A)$ and range $(A)$ denote the null space and the range space of a matrix $A$, respectively. The notation " $A \succeq 0$ " means that the symmetric matrix $A$ is positive semidefinite and " $A \succ 0$ " means that it is positive definite. For two symmetric matrices $A, B$ the notation " $A \succeq B$ " (" $A \succ B$ ") means that $A-B$ is positive semidefinite (positive definite). Finally, $x \neq 0$ means that at least one 
component of a vector $x$ is not equal to zero and $x>0$ says that all components of $x$ are greater than zero.

\section{Problem definition}

\subsection{Basic notations, generalized eigenvalues}

We consider a general mechanical structure, discrete or discretized by the finite element method. The number of members or finite elements is denoted by $m$, the total number of "free" degrees of freedom (i.e., not fixed by Dirichlet boundary conditions) by $n$. For a given set of $L$ (independent) load vectors

$$
f_{\ell} \in \mathbb{R}^{n}, \quad f_{\ell} \neq 0, \quad \ell=1, \ldots, L,
$$

the structure should satisfy linear equilibrium equations

$$
K(x) u_{\ell}=f_{\ell}, \quad \ell=1, \ldots, L .
$$

Here $K(x)$ is the stiffness matrix of the structure, depending on a design variable $x$. We will assume linear dependence of $K$ on $x$,

$$
K(x)=\sum_{i=1}^{m} x_{i} K_{i}
$$

with $x_{i} K_{i}$ being the element stiffness matrices. Note that the stiffness matrix of element (member) $e_{i}$ is typically defined as

$$
x_{i} K_{i}=x_{i} P_{i} \widehat{K}_{i} P_{i}^{T}
$$

where $P_{i} P_{i}^{T}$ is a projection from $\mathbb{R}^{n}$ to the space of element (member) degrees of freedom. In other words, $\widehat{K}_{i}$ is a matrix localized on the particular element, while $K_{i}$ lives in the full space $\mathbb{R}^{n}$. Further,

$$
x_{i} \widehat{K}_{i}=\int_{e_{i}} x_{i} B_{i}^{T} E_{i} B_{i} d V
$$

where the rectangular matrix $B_{i}$ contains derivatives of shape functions of the respective degrees of freedom and $E_{i}$ is a symmetric matrix containing information about material properties. To exclude pathological situations, we assume that

$$
f_{\ell} \in \operatorname{range}\left(\sum_{i=1}^{m} K_{i}\right) \quad \text { for all } \ell=1, \ldots, L
$$

which means that there exists a material distribution $x \geq 0$ that can carry all loads $f_{\ell}$, i.e., there exist corresponding $u_{1}, \ldots, u_{\ell}$ satisfying (2).

Similarly to the definition of $K(x)$, the mass matrix $M(x)$ of the structure is assumed to be given as

$$
M(x)=\sum_{i=1}^{m} x_{i} M_{i}, \quad M_{i}=P_{i} \widehat{M}_{i} P_{i}^{T}
$$

with element mass matrices

$$
x_{i} \widehat{M}_{i}=\int_{e_{i}} x_{i} N_{i}^{T} N_{i} d V
$$


Here $N_{i}$ contains the shape functions of the degrees of freedom associated with the $i^{\text {th }}$ element. The design variables $x \in \mathbb{R}^{m}, x \geq 0$, represent, for instance, the thickness, cross-sectional area or material properties of the element. We will assume that

$$
x_{i} \geq 0, \quad i=1, \ldots, m .
$$

Notice that the matrices $\widehat{K}_{i}, \widehat{M}_{i}$ have the properties $\widehat{K}_{i} \succeq 0, \widehat{M}_{i} \succ 0$, and thus $K(x) \succeq 0$, $M(x) \succeq 0$ for all $x \geq 0$. From a practical point of view, it is worth noticing that the element matrices $K_{i}$ and $M_{i}$ are very sparse with only nonzero elements corresponding to degrees of freedom of the $i^{\text {th }}$ element. That means, for each $i$, the matrices $K_{i}$ and $M_{i}$ have the same nonzero structure (see also Lemma 1 below). The matrices $K(x), M(x)$, however, may be dense, in general.

In the sequel, we will sometimes collect the displacement vectors $u_{1}, \ldots, u_{L}$ for all the load cases in one vector

$$
u=\left(u_{1}^{T}, \ldots, u_{L}^{T}\right)^{T} \in \mathbb{R}^{L \cdot n},
$$

for simplification of the notation.

In this paper we do not rely on any other properties of stiffness and mass matrices than those outlined above. Therefore, the problem formulations and the conclusions apply to a broad class of problems, e.g., to the variable thickness sheet problem or the free material optimization problem (see, e.g., [4]). For the sake of transparency, however, in the examples we concentrate on a particular class of discrete structures, namely trusses.

In this article, we will additionally consider free vibrations of the optimal structure. The free vibrations are the eigenvalues of the generalized eigenvalue problem

$$
K(x) w=\lambda\left(M(x)+M_{0}\right) w .
$$

The matrix $M_{0} \in \mathbb{R}^{n \times n}$ is assumed to be symmetric and positive semidefinite. It denotes the mass matrix of a given non-structural mass ("dead load"). The choice $M_{0}=0$ is of course included in our development.

In the sequel we use the notation

$$
X:=\left\{x \in \mathbb{R}^{m} \mid x \geq 0, x \neq 0\right\}
$$

for the set of all design variables referring to non-zero structures.

As a consequence of the construction of $K(x)$ and $M(x)$ we state a first fact which is widely known among practicioners. However, a strictly mathematical proof, although very simple, is difficult to find in the literature.

Lemma 1 For each $x \in X$ it holds that

$$
\operatorname{ker}\left(M(x)+M_{0}\right) \subseteq \operatorname{ker}(K(x))
$$

Proof: Let $u \in \mathbb{R}^{n}$ be in $\operatorname{ker}\left(M(x)+M_{0}\right)$. Then $u^{T}\left(M(x)+M_{0}\right) u=0$, i.e. (see (6)),

$$
0=u^{T}\left(\sum_{i=1}^{m} x_{i} P_{i} \widehat{M}_{i} P_{i}^{T}+M_{0}\right) u=\sum_{i=1}^{m} x_{i}\left(P_{i}^{T} u\right)^{T} \widehat{M}_{i}\left(P_{i}^{T} u\right)+u^{T} M_{0} u
$$


Because $\widehat{M}_{i} \succ 0$ for all $i$, and because $M_{0} \succeq 0$, we conclude that

$$
P_{i}^{T} u=0 \quad \text { for all } i \text { such that } x_{i}>0 .
$$

Hence, by the definition of $K(x)$ and by (4),

$$
K(x) u=\sum_{i=1}^{m} x_{i} K_{i} u=\sum_{i=1}^{m} x_{i} P_{i} \widehat{K}_{i} P_{i}^{T} u=\sum_{i: x_{i} \neq 0} x_{i} P_{i} \widehat{K}_{i} P_{i}^{T} u=0,
$$

and the proof is complete.

We now want to define a function $\lambda_{\min }(\cdot)$ that assigns a given structure represented by vector $x \in X$ the smallest eigenvalue $\lambda$ of problem (8). Before doing that, we mention the following dilemma hidden in the generalized eigenvalue problem (8). If $x \in X$ is fixed and $(\lambda, w) \in \mathbb{R} \times \mathbb{R}^{n}$ is a solution of (8) with $w \neq 0$ but with $w \in \operatorname{ker}\left(M(x)+M_{0}\right)$ then Lemma 1 shows that also $K(x) w=0$. Hence $(\mu, w)$ is also a solution of (8) for arbitrary $\mu \in \mathbb{R}$. In this situation we say that this eigenvalue is undefined; otherwise it is well-defined. Because undefined eigenvalues are meaningless from the engineering point of view, we want to exclude them from our considerations. This leads to the following notation.

Notation 2 For any $x \in X$, let $\lambda_{\min }(x)$ denote the smallest well-defined eigenvalue of (8), i.e.,

$$
\left.\begin{array}{ll}
\lambda_{\min }(x)=\min \left\{\lambda \mid \exists w \in \mathbb{R}^{n}:\right. & K(x) w=\lambda\left(M(x)+M_{0}\right) w, \\
& w \notin \operatorname{ker}\left(M(x)+M_{0}\right)
\end{array}\right\} .
$$

By standard linear algebra and by Lemma 1 it is seen that $\lambda_{\min }(x)$ can be written in the form of a Rayleigh quotient,

$$
\lambda_{\min }(x)=\inf _{u:\left(M(x)+M_{0}\right) u \neq 0} \frac{u^{T} K(x) u}{u^{T}\left(M(x)+M_{0}\right) u}
$$

for all $x \in X$ (see, e.g., [2] or [9]). This shows that the function $\lambda_{\min }(\cdot)$ is finite and nonnegative on $X$.

\subsection{Problem definition, difficulties}

Maximization of the smallest eigenvalue of a mechanical structure is of paramount importance in many industrial applications; see, e.g., [17]. In this article we define it as the problem of maximizing the smallest (well-defined) eigenvalue of (8) subject to equilibrium conditions and constraints on the compliance and on the volume:

$$
\max _{x \in \mathbb{R}^{m}, u \in \mathbb{R}^{L \cdot n}} \lambda_{\min }(x)
$$

subject to

$$
\begin{array}{ll}
\left(\sum_{i=1}^{m} x_{i} K_{i}\right) u_{\ell}=f_{\ell}, & \ell=1, \ldots, L \\
f_{\ell}^{T} u_{\ell} \leq \bar{\gamma}, & \ell=1, \ldots, L \\
\sum_{i=1}^{m} x_{i} \leq \bar{V} & \\
x_{i} \geq 0, & i=1, \ldots, m .
\end{array}
$$


This problem, or its minor modifications, has already been considered at several places in the literature. It finds valuable interest in practical applications (see $[17,19,13])$. To the knowledge of the authors, however, a rigorous treatment of this problem with positive semidefinite matrices $K$ and $M$ (i.e., permitting $x_{i}=0$ for some $i$, as needed in topology optimization) has not been considered, so far.

Remark 3 We mention that problem $(\mathrm{P})$ is closely related to the following minimum volume problem with an eigenvalue constraint:

$$
\begin{aligned}
& \min _{x \in \mathbb{R}^{m}, u \in \mathbb{R}^{L \cdot n}} \sum_{i=1}^{m} x_{i} \\
& \text { subject to } \\
& \begin{array}{ll}
\left(\sum_{i=1}^{m} x_{i} K_{i}\right) u_{\ell}=f_{\ell}, & \ell=1, \ldots, L \\
f_{\ell}^{T} u_{\ell} \leq \bar{\gamma}, & \ell=1, \ldots, L \\
\lambda_{\min }(x) \geq \bar{\lambda} & \\
x_{i} \geq 0, & i=1, \ldots, m .
\end{array}
\end{aligned}
$$

Here $\bar{\lambda}>0$ is a given lower bound for the minimal eigenvalue, and $\bar{\gamma}$ plays the same role as in problem $(\mathrm{P})$. Among practicioners, this problem is sometimes treated instead of $(\mathrm{P})$. It should be noted, however, that the solutions of both problems are generally not the same, even after a suitable scaling. An example is discussed below in Ex. 16.

Similarly, $(\mathrm{P})$ is closely related to the problem with the roles of $\lambda_{\min }(\cdot)$ and the function $(x, u) \mapsto \max _{1 \leq \ell \leq L} f_{\ell}^{T} u_{\ell}$ interchanged (the worst-case minimum compliance problem). Theoretical investigations on the interrelations between $(\mathrm{P}),(10)$, and the latter problem can be found in [2].

We now discuss several difficulties related to formulation $(\mathrm{P})$ in the light of its numerical treatment.

Difficulty 1: Nondifferentiability It is well-known that $\lambda_{\min }(\cdot)$ generally is a non-differentiable function. At least if $x>0$ it is easy to see that problem (8) is equivalent to

$$
\left(M(x)+M_{0}\right)^{-\frac{1}{2}} K(x)\left(M(x)+M_{0}\right)^{-\frac{1}{2}} w=\lambda w .
$$

Then $\lambda_{\min }(\cdot)$ is differentiable if the multiplicity of the minimal eigenvalue of the above problem is one (see, e.g., [13]). In numerical procedures, some practicioners circumvent nonsmoothness by small perturbations in the variable $x$ to achieve differentiability. It should be noted, however, that the use of algorithms of nonlinear (i.e., smooth) optimization in such a methodology may lead to wrong results.

Difficulty 2: Non-Lipschitzean behavior The natural cure to Difficulty 1 is the use of methods of Nonsmooth Optimization. These methods use generalized gradient information instead of gradient information, i.e., take non-smoothness into account. From the viewpoint of the authors the most general framework with yet numerically tractable problems is provided by the 


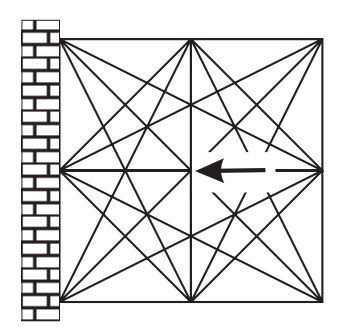

Figure 1: Example 4—initial design.

calculus of Clarke (see, e.g., [7]). This calculus works with functions that are locally Lipschitzcontinuous ("l.l.c." in short). A function $f: \tilde{X} \longrightarrow \mathbb{R}$ is defined to be 1.1.c. if for each $\bar{x} \in \tilde{X}$ there exists some neighborhood $U(\bar{x})$ of $\bar{x}$ and a constant $L=L(\bar{x})$ such that

$$
\frac{|f(x)-f(\bar{x})|}{\|x-\bar{x}\|} \leq L \quad \text { for all } x \in(\tilde{X} \cap U(\bar{x}))
$$

(where $\|\cdot\|$ denotes, e.g., the euclidean norm). Note that any 1.l.c. function is continuous. Property (11) shows that (maybe several distinct) limits of the quotient on the left hand side exist for $x \longrightarrow \bar{x}$. These limits then mimic the "slopes" of the non-smooth function $f$ at $\bar{x}$ when approaching $\bar{x}$ from different directions, say. These data can be used also in a numerical approach, e.g., building a piece-wise linear model near $\bar{x}$. There exist a few algorithms and codes for calculating a local optimizer of a 1.l.c. function $f$.

Hence, if $\lambda_{\min }(\cdot)$ was 1.l.c., then the nonsmooth calculus of Clarke could be used and known numerical procedures could tackle problem $(\mathrm{P})$. The following numerical example, however, indicates that $\lambda_{\min }(\cdot)$ lacks to be 1.1.c. near the boundary of $X$. This is slightly unexpected, given the well-known fact that the eigenvalues of the standard symmetric eigenvalue problem are 1.1.c. functions (see, e.g., [5]).

Example 4 Consider a $3 \times 3$ ground-structure on a square $1 \times 1$ area in $2 \mathrm{D}$ with all nodes connected and with a horizontal force $(-1,0)$ applied at the central node $(L=1)$; see Fig. 1 . We use the (scaled) Young's modulus 1.0, for simplicity, in all bars. Now consider problem (P) where we have replaced the zero lower bound on the design variables by a parameter $\varepsilon \geq 0$.

$$
\begin{array}{ll}
\max _{\substack{x \in \mathbb{R}^{m}, u \in \mathbb{R}^{L} \\
\text { subject to }}} \lambda_{\min }(x) & \\
& \left(\sum_{i=1}^{m} x_{i} K_{i}\right) u_{\ell}=f_{\ell}, \quad \ell=1, \ldots, L \\
f_{\ell}^{T} u_{\ell} \leq \bar{\gamma}, & \ell=1, \ldots, L \\
\sum_{i=1}^{m} x_{i} \leq \bar{V} & \\
x_{i} \geq \varepsilon, & i=1, \ldots, m .
\end{array}
$$

Let $\left(x_{\varepsilon}^{*}, u_{\varepsilon}^{*}\right)$ denote a solution of this problem. We have numerically calculated $\left(x_{\varepsilon}^{*}, u_{\varepsilon}^{*}\right)$ for $1.5 \cdot 10^{-7} \leq \varepsilon \leq 2 \cdot 10^{-3}$ (We will show later on how this can be done). Figure 2 shows the 

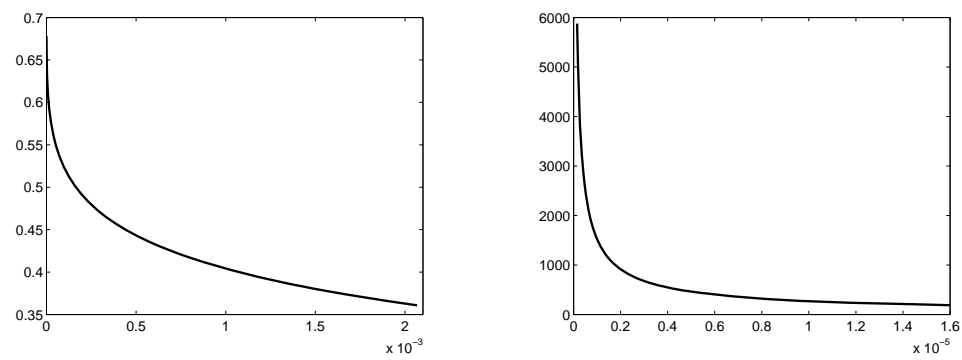

Figure 2: Example 4 demonstrating apparent non-Lipschitz behavior of the minimum eigenvalue function close to the boundary of the feasible region. The graph of the function (left) and its derivative (right) are shown.
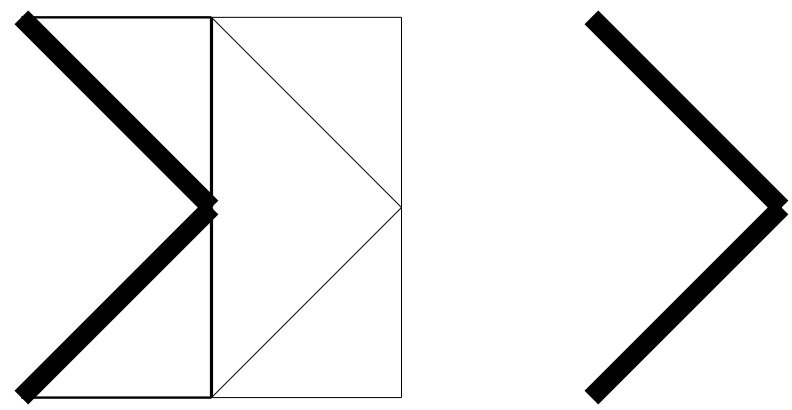

Figure 3: Example 4—optimal structures for $x_{i} \geq 2 \cdot 10^{-3}$ (left) and $x_{i} \geq 0$ (right).

behavior of the objective function $\lambda_{\min }(\cdot)$ at the optimizers $x_{\varepsilon}^{*}$ when $\varepsilon$ is varied. The function $\lambda_{\min }(\cdot)$ looks all but Lipschitz (for smaller values of $\varepsilon$ we were unable to compute the function value due to round-off errors). To see its behavior more clearly, we plot in the right-hand figure the derivative (computed by finite differences) in the interval $\left[1.5 \cdot 10^{-7}, 1.6 \cdot 10^{-5}\right]$; this figure confirms the non-Lipschitz behavior. When we solve the minimum eigenvalue problem (P) with $x \geq 0$, we obtain the optimum value $\lambda^{*}=0.7071068$. Obviously, the picture is not a proof of a non-Lipschitz behavior but it is very indicative. The optimal trusses for $\varepsilon=2 \cdot 10^{-3}$ and for the problem with $x \geq 0$ are shown in Figure 3 (left and right, respectively). In the first case, only bars that are not equal to the lower bound $\varepsilon$ are presented. In both cases, the compliance constraint was inactive.

The use of positive lower bounds is also addressed below.

Difficulty 3: Discontinuity As already indicated in the previous example, problem $(\mathrm{P})$ inherently contains an even more serious difficulty which is not seen at a first glance. First, it can be proved that $\lambda_{\min }(\cdot)$ is upper semicontinuous ("u.s.c." in short) on $X$, i.e., that for each sequence $\left(x^{i}\right)_{i \in \mathbb{N}} \subset X$ of structures converging to some structure $\bar{x} \in X$ we have the inequality

$$
\limsup _{i \rightarrow \infty} \lambda_{\min }\left(x^{i}\right) \leq \lambda_{\min }(\bar{x})
$$

(for the proof, see [2]). Unfortunately, lower semicontinuity, i.e.,

$$
\liminf _{i \rightarrow \infty} \lambda_{\min }\left(x^{i}\right) \geq \lambda_{\min }(\bar{x}),
$$




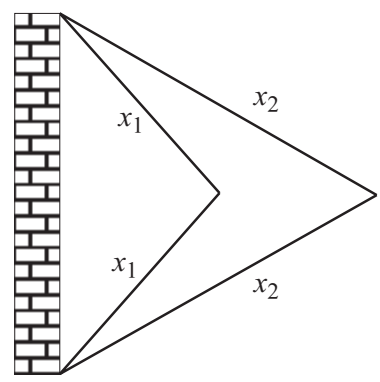

Figure 4: Example showing possible discontinuity of $\lambda_{\min }$

does not necessarily hold for structures $\bar{x}$ on the boundary of $X$. As a consequence, $\lambda_{\min }(\cdot)$ lacks to be continuous at such points. This unpleasant fact is related to the situation that some eigenvalues may become undefined when $K(\bar{x})$ becomes singular. The following example of academic size illustrates this behavior.

Example 5 Consider the planar truss depicted in Figure 4 with the four nodal points $(0,0)$, $(0,1),\left(\frac{1}{2}, \frac{1}{2}\right)$, and $\left(1, \frac{1}{2}\right)$. Let the truss be symmetric w.r.t. its horizontal axis, so consider only two design variables, $x_{1}$ and $x_{2}$, denoting bar volumes. Again, the Young's modulus is 1.0 in all bars, for simplicity. Then the corresponding stiffness and mass matrix have the following form.

$$
\begin{aligned}
K(x) & =\left(\begin{array}{cccc}
x_{1} \cdot 2 & 0 & 0 & 0 \\
0 & x_{1} \cdot 2 & 0 & 0 \\
0 & 0 & x_{2} \cdot \frac{32}{25} & 0 \\
0 & 0 & 0 & x_{2} \cdot \frac{8}{25}
\end{array}\right) \\
M(x) & =\left(\begin{array}{cccc}
x_{1} \cdot 2 \sqrt{2} & 0 & 0 & 0 \\
0 & x_{1} \cdot 2 \sqrt{2} & 0 & 0 \\
0 & 0 & x_{2} \cdot 2 \sqrt{5} & 0 \\
0 & 0 & 0 & x_{2} \cdot 2 \sqrt{5}
\end{array}\right)
\end{aligned}
$$

Hence, if $x_{1}>0$ and $x_{2}>0$ then, due to the special situation that here $K$ and $M$ are diagonal, we can easily calculate the four structural eigenvalues by taking the quotients of corresponding entries in the diagonals of $K$ and $M$,

$$
\begin{array}{ll}
\frac{2}{2 \sqrt{2}} \frac{x_{1}}{x_{1}}=\frac{1}{\sqrt{2}} \approx 0.71, & \frac{2}{2 \sqrt{2}} \frac{x_{1}}{x_{1}}=\frac{1}{\sqrt{2}} \approx 0.71, \\
\frac{32}{25 \cdot 2 \sqrt{5}} \frac{x_{2}}{x_{2}} \approx 0.29, & \frac{8}{25 \cdot 2 \sqrt{5}} \frac{x_{2}}{x_{2}} \approx 0.07 .
\end{array}
$$

Analogously, if $x_{1}>0$ and $x_{2}=0$ then the first two eigenvalues are $\frac{2}{2 \sqrt{2}} \frac{x_{1}}{x_{1}} \approx 0.71$ as before, but the remaining two eigenvalues become undefined. Summarizing, for any design vector $x=\left(x_{1}, x_{2}\right)$ with $x_{1}>0$ we obtain

$$
\lambda_{\min }(x)= \begin{cases}\frac{8}{50 \sqrt{5}} \approx 0.07 & \text { for } x_{2}>0 \\ \frac{1}{\sqrt{2}} \approx 0.71 & \text { for } x_{2}=0 .\end{cases}
$$

Thus, $\lambda_{\min }(\cdot)$ is discontinuous at all points $x$ with $x_{1}>0$ and $x_{2}=0$. As seen, the reason for the discontinuity lies in the fact that, when $x_{2}=0$, the eigenvalue $\frac{8}{50 \sqrt{5}} \frac{x_{2}}{x_{2}}$ becomes undefined 

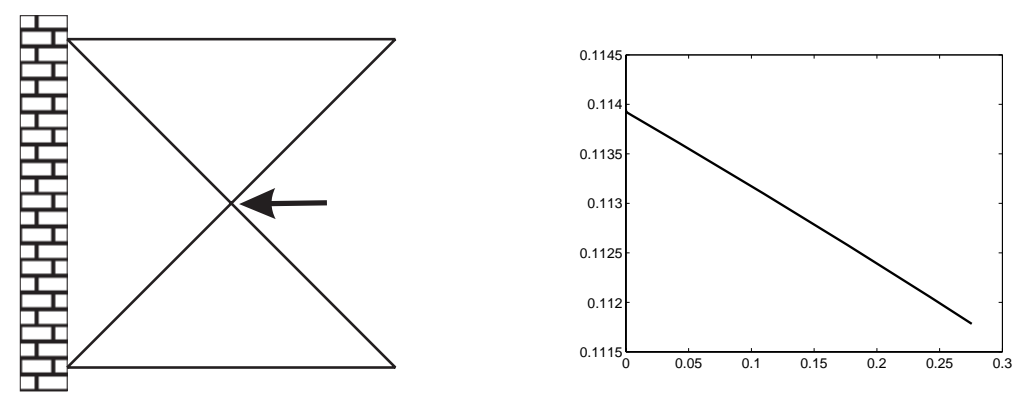

Figure 5: Example 6 demonstrating possible discontinuity of $\lambda_{\min }$; initial structure (left) and graph of the minimal eigenvalue as a function of $\varepsilon$ (right).

and $\lambda_{\min }(\cdot)$ "jumps up" to what was before the second smallest eigenvalue. This example also nicely illustrates the upper semi-continuity of $\lambda_{\min }(\cdot)$ mentioned above; see (12).

Note that the possible discontinuity of $\lambda_{\min }(\cdot)$ prevents the use of the continuation approach already discussed above, i.e., the use of smaller and smaller positive lower bounds on the design variables (see problem $\left(\mathrm{P}_{\varepsilon}\right)$ ). This continuation approach is widely used in Structural Optimization. To make this transparent, let again $\left(x_{\varepsilon}^{*}, u_{\varepsilon}^{*}\right)$ denote a solution of problem $\left(\mathrm{P}_{\varepsilon}\right)$ for each $\varepsilon \in[0, \delta]$ (where $\delta>0$ is some given number). Assume that the solution structure $x_{\varepsilon}^{*}$ is unique for all $\varepsilon \in[0, \delta]$, and that there exists $\bar{x}$ with $x_{\varepsilon}^{*} \longrightarrow \bar{x}$ for $\varepsilon \searrow 0$. Then, due to possible discontinuity, it might happen that

$$
\lim _{\varepsilon \searrow 0} \lambda_{\min }\left(x_{\varepsilon}^{*}\right)<\lambda_{\min }(\bar{x})<\lambda_{\min }\left(x_{0}^{*}\right),
$$

i.e., the limiting structure $\bar{x}$ is different from the solution structure of the problem $\left(\mathrm{P}_{\varepsilon}\right)$ for $\varepsilon=0$, the unperturbed problem $(\mathrm{P})$.

The following example shows that $\lambda_{\min }\left(x_{\varepsilon}^{*}\right)$ for $\varepsilon \searrow 0$ may converge to a value that is far below the true optimal value $\lambda_{\min }\left(x^{*}\right)$ of $(\mathrm{P})$ (although here $x_{\varepsilon}^{*} \rightarrow x_{0}^{*}$ ).

Example 6 Consider the 2D ground-structure shown in Figure 5, together with boundary condition and the force applied at the central node. Figure 5-right shows the behavior of the objective function $\lambda_{\min }\left(x_{\varepsilon}^{*}\right)$ of the problem $\left(\mathrm{P}_{\varepsilon}\right)$, where $\varepsilon$ lies in the interval $\left[2 \cdot 10^{-5}, 3 \cdot 10^{-3}\right]$. We can observe linear behavior of the minimal eigenvalue; this (multiple) eigenvalue is associated with vibrations of the right-hand corners that are only connected to the structure by the $\varepsilon-$ thick bars (see Figure 6-left). Hence, when $\varepsilon$ reaches zero, those bars disappear, the corresponding entries in the stiffness and mass matrices become zero and the eigenvalue becomes undefined. Using the continuation approach, we would then use a limit of the sequence of solutions for $\varepsilon \rightarrow 0$, i.e., we arrive at a value around 0.113924 for $\lambda_{\min }\left(x^{*}\right)$ (which is $\lambda_{\min }\left(x_{\varepsilon}^{*}\right)$ for $\varepsilon=2 \cdot 10^{-5}$ ). However, solving problem $(\mathrm{P})$, i.e., $\left(\mathrm{P}_{\varepsilon}\right)$ with $\varepsilon=0$ (see below how to do this...) then we obtain the true solution $x^{*}$ of $(\mathrm{P})$ with $\lambda_{\min }\left(x^{*}\right)=0.707107$; the optimal structure is depicted in Figure 6-right. Here we clearly see the discontinuity of $\lambda_{\min }$ on the boundary of the feasible domain. 

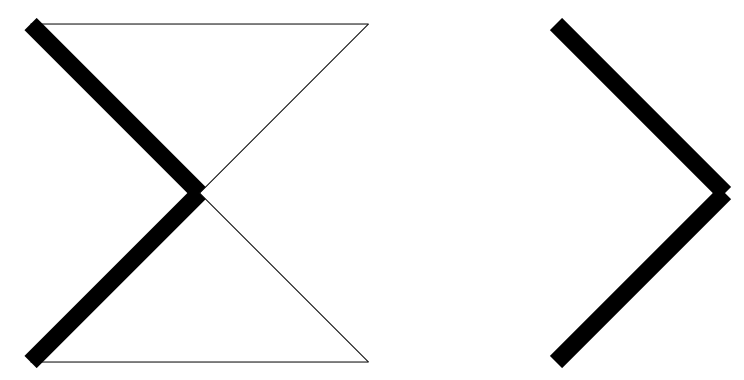

Figure 6: Example 6—optimal structures for $x_{i} \geq 2 \cdot 10^{-5}$ (left) and $x_{i} \geq 0$ (right).

Difficulties 4 to 6 There are three other (though minor) difficulties in the numerical approach dealing with $(\mathrm{P})$. First, problem $(\mathrm{P})$ is nonconvex and thus we cannot expect more than local optimality of a solution obtained by a suitable numerical approach, whether is this approach based on descent concepts or on optimality conditions (due to the large number of variables, application of methods from global optimization are not applicable in practical situations). Second, the numerical calculation of $\lambda_{\min }(x)$ at given points $x$ is expensive and delicate. Although there are well-established numerical procedures in program libraries to solve this problem, it is still a challenge to calculate $\lambda_{\min }(x)$ in reasonable time and with sufficient precision. The same is true for the calculation of a corresponding eigenvector which is needed for the calculation of the gradient of $\lambda_{\min }$ at $x$ (provided $\lambda_{\min }$ is differentiable at $x$ ). There are three other troubles connected to this point. First, only the minimal eigenvalue should be calculated. Due to the size of the matrix, it is not desirable to calculate all eigenvalues and select the minimal one. Second, accuracy is a problem, in particular for the decision whether an eigenvalue is the minimal one, whether its multiplicity is one or bigger, and whether it is well-defined at all. This corresponds to the third trouble, ill-conditioning: if $K(x)$ is (nearly) singular (and this is often the case in topology optimization), most of the solution procedures will break down.

As a consequence of all these difficulties, we conclude that formulation (P) is not useful for our purpose, i.e., numerical solution of the topology optimization problem. From the authors' point of view, the most crucial obstacles are the non-Lipschitzean behavior and the discontinuity of the objective function because they are of theoretical nature and exclude the use of standard numerical procedures. Notice that in meaningful topology optimization problems many or even most of the design variables $x_{i}$ will become zero at the optimum and thus the treatment of singular stiffness matrices and the related non-Lipschitzean behavior or discontinuity of $\lambda_{\min }(\cdot)$ is a must.

In the following section we present an equivalent formulation of the problem which largely overcomes all the difficulties explained above.

\section{Reformulation as semidefinite program}

Recall that problem (P) is nonconvex and discontinuous. Furthermore, it implicitly includes the computation of the smallest eigenvalue $\lambda_{\min }\left(x^{k}\right)$ of (8) at each iteration point $x^{k}$ of a certain solution procedure. In this section we give a reformulation of $(\mathrm{P})$ which is much easier to analyze and to solve numerically. Although this reformulation seems to be known in the com- 
munity of Mathematical Programming, it has never been used for the numerical solution, up to our knowledge.

We start with an auxiliary result expressing the compliance constraints as so-called linear matrix equalities based on the ordering cone of positive semidefinite matrices. Recall the notation " $\succeq$ " explained at the end of the Introduction.

Proposition 7 Let $x \in \mathbb{R}^{m}, x \geq 0$, and $\gamma \in \mathbb{R}$ be fixed, and fix an index $\ell \in\{1, \ldots, L\}$. Then there exists $u_{\ell} \in \mathbb{R}^{n}$ satisfying

$$
K(x) u_{\ell}=f_{\ell} \quad \text { and } \quad f_{\ell}^{T} u_{\ell} \leq \gamma
$$

if and only if

$$
\left(\begin{array}{cc}
\gamma & -f_{\ell}^{T} \\
-f_{\ell} & K(x)
\end{array}\right) \succeq 0
$$

Proof: Note that $K(x)$ may be singular in our case, so that we cannot directly use the Schur complement theorem (see, e.g., [3]). We first write the matrix inequality equivalently as

$$
\alpha^{2} \gamma-2 \alpha f_{\ell}^{T} v+v^{T} K(x) v \geq 0 \quad \forall \alpha \in \mathbb{R}, \forall v \in \mathbb{R}^{n} .
$$

" $\Rightarrow$ " As $K(x) \succeq 0$, we know that $u_{\ell}$ minimizes the quadratic functional $\left(v \mapsto v^{T} K(x) v-\right.$ $\left.2 f_{\ell}^{T} v\right)$ with the minimal value $-f_{\ell}^{T} u_{\ell}$. Thus

$$
v^{T} K(x) v-2 f_{\ell}^{T} v \geq-f_{\ell}^{T} u_{\ell} \geq-\gamma \quad \forall v \in \mathbb{R}^{n} .
$$

Using the substitution $v=\sigma w, \sigma \in \mathbb{R}$, we can write this as

$$
(\sigma w)^{T} K(x)(\sigma w)-2 f_{\ell}^{T}(\sigma w) \geq-\gamma \quad \forall \sigma \in \mathbb{R}, \forall w \in \mathbb{R}^{n},
$$

hence

$$
w^{T} K(x) w-\frac{1}{\sigma} 2 f_{\ell}^{T} w \geq-\frac{1}{\sigma^{2}} \gamma \quad \forall \sigma \in \mathbb{R} \backslash\{0\}, \forall w \in \mathbb{R}^{n}
$$

which is just (13) with $\alpha=\frac{1}{\sigma}$.

" $\Leftarrow$ " Put $\alpha=1$. Then we get from (13) that

$$
\gamma-2 f_{\ell}^{T} v+v^{T} K(x) v \geq 0 \quad \forall v \in \mathbb{R}^{n},
$$

and so the convex quadratic function

$$
q: v \mapsto-2 f_{\ell}^{T} v+v^{T} K(x) v
$$

is bounded from below. By this, standard linear algebra shows that $q(\cdot)$ possesses a global minimizer $u_{\ell} \in \mathbb{R}^{n}$, i.e., the gradient of $q$ vanishes at $u_{\ell}$, proving

$$
K(x) u_{\ell}=f_{\ell} .
$$

Inserting this into (13) with $\alpha=1$, we have

$$
\gamma-2 f_{\ell}^{T} u_{\ell}+u_{\ell}^{T} f_{\ell} \geq 0
$$

that is, $\gamma \geq f_{\ell}^{T} u_{\ell}$, and we are done.

As a second step we use a different representation of $\lambda_{\min }(\cdot)$, based again on matrix inequalities. 
Proposition 8 For all $x \in X$,

$$
\lambda_{\min }(x)=\sup \left\{\lambda \mid K(x)-\lambda\left(M(x)+M_{0}\right) \succeq 0\right\} .
$$

Proof: Let $x \in X$ be given and recall representation (9) of $\lambda_{\min }(x)$.

Let us first show the " $\geq$ " part. Take an arbitrary $\lambda$ satisfying $K(x)-\lambda\left(M(x)+M_{0}\right) \succeq 0$, i.e.,

$$
u^{T} K(x) u-\lambda u^{T}\left(M(x)+M_{0}\right) u \geq 0 \quad \forall u \neq 0 .
$$

Consider $u$ with $\left(M(x)+M_{0}\right) u \neq 0$; then we have

$$
\frac{u^{T} K(x) u}{u^{T}\left(M(x)+M_{0}\right) u} \geq \lambda
$$

Because $\lambda$ and $u$ were arbitrary, we can write "inf" in front of the fraction and "sup" in front of $\lambda$ and the inequality remains valid. Now insert (9).

The proof of the " $\leq$ " part is similar: Let

$$
\tilde{\lambda}:=\inf _{u:\left(M(x)+M_{0}\right) u \neq 0} \frac{u^{T} K(x) u}{u^{T}\left(M(x)+M_{0}\right) u} .
$$

Then

$$
\tilde{\lambda} \leq \frac{u^{T} K(x) u}{u^{T}\left(M(x)+M_{0}\right) u}
$$

for all $u$ with $\left(M(x)+M_{0}\right) u \neq 0$ which in turn means that

$$
u^{T} K u-\tilde{\lambda} u^{T}\left(M(x)+M_{0}\right) u \geq 0 \quad \forall u:\left(M(x)+M_{0}\right) u \neq 0 .
$$

If $\left(M(x)+M_{0}\right) u=0$ then $u \in \operatorname{ker}(K(x))$ by Lemma 1 , and thus the above inequality holds as well. All in all, $K(x)-\tilde{\lambda}\left(M(x)+M_{0}\right) \succeq 0$, i.e.,

$$
\tilde{\lambda} \leq \sup \left\{\lambda \mid K(x)-\lambda\left(M(x)+M_{0}\right) \succeq 0\right\} .
$$

Proposition 7 shows that the displacement vectors $u_{\ell}$ may be eliminated and that the compliance constraints may be treated by matrix inequalities which linearly depend on the design variable $x$. Similarly, Prop. 8 shows that $\lambda_{\min }(\cdot)$ may be expressed through a variable $\lambda \in \mathbb{R}$ subject to matrix inequality as constraints. We arrive at the following problem formulation:

$$
\max _{x \in \mathbb{R}^{m}, \lambda \in \mathbb{R}} \lambda
$$

subject to

$$
\begin{array}{ll}
\left(\begin{array}{cc}
\bar{\gamma} & -f_{\ell}^{T} \\
-f_{\ell} & K(x)
\end{array}\right) \succeq 0, & \ell=1, \ldots, L \\
\sum_{i=1}^{m} x_{i} \leq \bar{V} & \\
x_{i} \geq 0, & i=1, \ldots, m \\
K(x)-\lambda\left(M(x)+M_{0}\right) \succeq 0 . &
\end{array}
$$


Here the constants $\bar{\gamma}$ and $\bar{V}$ are given as in the original problem formulation (P). Notice that the variables are now $x$ (as before) and $\lambda \in \mathbb{R}$ (new). The state variables $u_{\ell}, \ell=1, \ldots, L$, have been eliminated and are implicitly hidden in the first group of matrix inequalities.

Due to the matrix inequalities among the constraints, problem $(\mathrm{S})$ belons to the class of so-called semidefinite programming problems (SDP). During the past decade this problem class has been extensively studied by many researchers of the mathematical programming community. For introduction to SDPs we refer to the monographies [3] and [8].

The above problem reformulation results in the following theorem. It directly follows from Propositions 7 and 8.

Theorem 9 (a) If $\left(x^{*}, u^{*}\right)$ is a global maximizer of $(\mathrm{P})$ then $\left(x^{*}, \lambda^{*}\right)$ is a global maximizer of $(\mathrm{S})$ and $\lambda^{*}:=\lambda_{\min }\left(x^{*}\right)$. Moreover, the optimal values of both problems coincide.

(b) If $\left(x^{*}, \lambda^{*}\right)$ is a global maximizer of $(\mathrm{S})$ then there exists $u^{*}$ such that $\left(x^{*}, u^{*}\right)$ is a global maximizer of $(\mathrm{P})$. Moreover, the optimal values of both problems coincide, i.e., $\lambda^{*}=$ $\lambda_{\min }\left(x^{*}\right)$.

We emphasize that, due to the SDP reformulation, the originally discontinuous problems became continuous; a fact of big practical value. Moreover, the numerically difficult evaluation of $\lambda_{\min }(\cdot)$ is circumvented, but matrix inequalities must be treated instead. Also note that $(\mathrm{S})$ is an SDP problem with a bilinear matrix inequality (BMI) constraint, i.e., is generally nonconvex. We remark, however, that problem (S) hides a quasiconvex structure; see [2].

By using the Propositions 7 and 8, we may also clarify the existence of solutions of our problems.

Theorem 10 Problem (S) (or, equivalently, problem $(\mathrm{P})$ ) possesses a solution if and only if it possesses feasible points.

Proof: By Prop. 7, problem (S) can be written in the form

$$
\max \left\{\lambda_{\min }(x) \mid x \in \mathcal{F}\right\}
$$

with the feasible set

$$
\mathcal{F}:=\left\{x \in \mathbb{R}^{m} \mid\left(\begin{array}{rc}
\bar{\gamma} & -f_{\ell}^{T} \\
-f_{\ell} & K(x)
\end{array}\right) \succeq 0 \forall \ell ; x \geq 0 ; \sum_{i=1}^{m} x_{i} \leq \bar{V}\right\} .
$$

Because the cone of positive semidefinite matrices is closed, the set $\mathcal{F}$ is compact. Moreover, $0 \notin \mathcal{F}$ due to assumption (1), i.e., $\mathcal{F} \subset X$. Hence, because $\lambda_{\min }(\cdot)$ is upper semi-continuous (u.s.c.) on $X$ (see, e.g., [2]), it is u.s.c. on $\mathcal{F}$. Now, each u.s.c. function attains its supremum on a non-empty compact set (see, e.g., [15, Thm. 2.13.1]).

\section{Calculation of global maximizers}

Instead of using methods from Global optimization for the calculation of a global maximizer of problem (S), we may use the close relation of (S) to certain convex SDPs. In the following we 
propose a practical framework for finding the global solution of (S) (or (P); see Thm. 9) based on the solutions of a sequence of convex SDPs.

For fixed $\lambda \geq 0$ and fixed $\delta \geq 0$ consider the following linear SDP:

$$
\begin{aligned}
& \min _{x \in \mathbb{R}^{m}, V \in \mathbb{R}} V \\
& \text { subject to } \\
& \left.\qquad \begin{array}{cc}
\bar{\gamma} & -f_{\ell}^{T} \\
-f_{\ell} & K(x)
\end{array}\right) \succeq 0, \quad \ell=1, \ldots, L \\
& \quad \sum_{i=1}^{m} x_{i} \leq V \\
& V \leq \bar{V} \\
& x_{i} \geq 0, \\
& K(x)-(\lambda+\delta)\left(M(x)+M_{0}\right) \succeq 0 .
\end{aligned}
$$$$
\left(\mathrm{S}_{\mathrm{vol}}[\lambda, \delta]\right)
$$

We mention that an SDP of this type has first been formulated and studied in [16]. It represents a problem where volume is minimized subject to compliance constraints (see Prop. 7) and eigenvalue constraints. Note that by Prop. 8 the matrix inequality constraint

$$
K(x)-(\lambda+\delta)\left(M(x)+M_{0}\right) \succeq 0
$$

simply means that $\lambda_{\min }(x) \geq \lambda+\delta$. Problem $\left(\mathrm{S}_{\mathrm{vol}}[\lambda, \delta]\right)$ is therefore just an extension of the problem (10) mentioned above in Remark 3.

In the following, the feasible set of problem $\left(\mathrm{S}_{\mathrm{vol}}[\lambda, \delta]\right)$ is denoted by $\mathcal{F}(\lambda, \delta)$, for simplicity. Notice that $\left(\mathrm{S}_{\mathrm{vol}}[\lambda, \delta]\right)$ is a linear SDP, i.e., a convex optimization problem for which a global maximizer can be calculated, provided $\mathcal{F}(\lambda, \delta) \neq \varnothing$. Moreover, because $\left(\mathrm{S}_{\text {vol }}[\lambda, \delta]\right)$ is a convex SDP, modern solution procedures are able to recognize (up to numerical acurracy) whether $\mathcal{F}(\lambda, \delta)=\varnothing$ or not.

The following proposition gives a tool for the estimation of the (globally) optimal objective function value of problem $(\mathrm{S})$. Its proof is easy because the constraints in the considered problems are almost identical.

Proposition 11 Let $(\tilde{x}, \lambda)$ be feasible for $(\mathrm{S})$ and let $\lambda^{* *}$ denote the (globally) optimal function value of problem (S). Moreover, let $\delta>0$ be arbitrary and consider the problem $\left(\mathrm{S}_{\mathrm{vol}}[\lambda, \delta]\right)$ with the parameters $\bar{\gamma}$ and $\bar{V}$ copied from (S). Then the following assertions hold:

(a) If $\mathcal{F}(\lambda, \delta) \neq \varnothing$ then for each $(x, V) \in \mathcal{F}(\lambda, \delta)$ the point $(x, \lambda+\delta)$ is feasible for (S), i.e.,

$$
\lambda<\lambda+\delta \leq \lambda^{* *} .
$$

(b) If $\mathcal{F}(\lambda, \delta)=\varnothing$ then

$$
\lambda \leq \lambda^{* *}<\lambda+\delta
$$

The practical value of this proposition lies in the possibility to improve upper and lower bounds for $\lambda^{* *}$ which can be numerically calculated through solutions (or only feasible points) of the convex linear SDPs of the type $\left(\mathrm{S}_{\mathrm{vol}}[\lambda, \delta]\right)$. 
As a pre-processing step, we first calculate initial lower and upper bounds $\lambda_{0}^{L}, \lambda_{0}^{U}$ on $\lambda^{* *}$. For this, first compute a feasible point $(x, \lambda)$ of (S) and choose arbitrary $\bar{\delta}>0$. Then find the smallest $k \in \mathbb{N}$ such that $\mathcal{F}\left(\lambda, 2^{k} \bar{\delta}\right)=\varnothing$ by treating $\left(\mathrm{S}_{\mathrm{vol}}\left[\lambda, 2^{k} \bar{\delta}\right]\right)$ repeatedly. Set

$$
\lambda_{0}^{L}:=\lambda+2^{k-1} \bar{\delta} \quad \text { and } \quad \lambda_{0}^{U}:=\lambda+2^{k} \bar{\delta} .
$$

Then Prop. 11 shows that

$$
0 \leq \lambda_{0}^{L} \leq \lambda^{* *}<\lambda_{0}^{U}
$$

With these bounds it is easy to construct the following bisection type algorithm which in each step reduces the gap $\left(\lambda_{k}^{U}-\lambda_{k}^{L}\right)$ by a factor of (at least) $\frac{1}{2}$.

Algorithm 12 Choose an accuracy $\eta>0$ and a feasible point $\left(x_{0}, \lambda_{0}\right)$ for (S).

Put $\delta_{0}:=\frac{1}{2}\left(\lambda_{0}^{U}-\lambda_{0}^{L}\right)$ and $k:=0$. Go to Step 2 .

1. Calculate a feasible point [or even a local maximizer] $\left(x_{k}, \lambda_{k}\right)$ of (S) with the additional constraint " $\lambda \geq \lambda_{k}^{L}$ ".

2. If $\lambda_{k}>\lambda_{k}^{L}$ then update $\lambda_{k}^{L}$ by $\lambda_{k}^{L}:=\lambda_{k}$.

3. If $\lambda_{k}^{U}-\lambda_{k}^{L} \leq \eta$ then EXIT with the result $\left(x^{*}, \lambda^{*}\right):=\left(x_{k}, \lambda_{k}\right)$.

4. Put $\delta_{k}:=\frac{1}{2}\left(\lambda_{k}^{U}-\lambda_{k}^{L}\right)$ and consider problem $\left(\mathrm{S}_{\mathrm{vol}}\left[\lambda_{k}, \delta_{k}\right]\right)$.

If $\mathcal{F}\left(\lambda_{k}, \delta_{k}\right) \neq \varnothing$ then:

4A. Put $\lambda_{k+1}^{L}:=\lambda_{k}^{L}+\delta_{k}, k:=k+1$, and go to Step 1 .

Otherwise, if $\mathcal{F}\left(\lambda_{k}, \delta_{k}\right)=\varnothing$, then:

4B. Put $\lambda_{k+1}^{U}:=\lambda_{k}^{U}-\delta_{k}, k:=k+1$, and go to Step 1 .

The proof of the following proposition is a straightforward exercise.

Proposition 13 Let $(\mathrm{S})$ possess a global solution $\left(x^{* *}, \lambda^{* *}\right)$ (see Thm. 10). Then the following assertions hold.

(a) Algorithm 12 is well-defined, and after each iteration we have

$$
\lambda_{k}^{L} \leq \lambda_{k} \leq \lambda^{* *}<\lambda_{k}^{U} \quad \text { and } \quad \lambda_{k}^{U}-\lambda_{k}^{L} \leq 2^{-k}\left(\lambda_{0}^{U}-\lambda_{0}^{L}\right) .
$$

(b) Algorithm 12 terminates after a finite number $K$ of iterations, and

$$
K \leq\left\lceil\frac{\ln \left(\lambda_{0}^{U}-\lambda_{0}^{L}\right)-\ln (\eta)}{\ln (2)}\right\rceil
$$

(where $\lceil\alpha\rceil=\min \{N \mid N \in \mathbb{N}, \alpha \leq N\}$, as usual).

At termination, the result $\left(x^{*}, \lambda^{*}\right)$ is feasible for $(\mathrm{S})$ with

$$
\lambda^{* *}-\lambda^{*} \leq \eta \text {. }
$$


Notice that the additional constraint " $\lambda \geq \lambda_{k}^{L}$ " in Step 1 of Algorithm 12 does not cause any trouble because it is linear. But it guarantees that the sequence $\left(\lambda_{k}\right)_{k}$ is monotonically increasing. Moreover, the calculation of global maximizers (in Step 4A), resp. local maximizers (in Step 1), instead of just feasible points should significantly speed up the algorithm. In this case the update of $\lambda_{k}^{U}$ in Step 4B, resp. of $\lambda_{k}^{L}$ in Step 2, may lead to a much bigger reduction of the gap $\lambda_{k}^{U}-\lambda_{k}^{L}$.

For the numerical treatment of the SDP problems (S) and $\left(\mathrm{S}_{\mathrm{vol}}[\lambda, \delta]\right)$ one must resort to methods of semidefinite programming. Such methods, and corresponding codes, are nowadays available for linear SDPs. We mention Internet pages plato.la.asu.edu/bench.html which includes the list of available SDP solvers and also benchmarks of SDP software and neos.mcs.anl.gov/neos/solvers/ which can be used for on-line solution of SDP problems. The limiting factor of these codes is, however, the problem size which, compared to general nonlinear programs, is restricted to problems of medium size. The problem $(\mathrm{S})$ even requires a method which can deal with bilinear matrix inequalities. We will use such a method to solve example problems in the next section. It should be noted, however, that algorithms and codes for SDPs with bilinear matrix inequalities are on the edge of current research and are not yet standard.

\section{Numerical examples}

In this chapter we present numerical examples which will, on the one hand, illustrate some of the theoretical results and, on the other hand, demonstrate the practical use of the SDP problem formulation.

The code we have used for the solution of the nonlinear SDP formulations is PENBMI, version 2.1 (see [12]). This code implements the generalized Augmented Lagrangian method, as described in $[11,21]$. In particular, PENBMI can treat bilinear matrix inequalities as is necessary for problem $(\mathrm{S})$ (see [10]).

The examples were solved on a Pentium 4-M 2GHz PC running Windows XP. All problems were formulated and solved in MATLAB using the YALMIP parser [14] to PENBMI. Apart from the CPU time needed to solve the examples, we will also give the number of inner iterations of PENBMI. One inner iterations basically amounts to the solution of a system of linear equations of dimension $m$.

Example 14 Consider a 3-by-3 truss with all nodes connected by potential bars. The nodes on the left-hand side are fixed in both directions, a horizontal force $(-1,0)$ is applied at the right-middle node; see Figure 7-left. No nonstructural mass is considered, i.e., $M_{0}=0$. The Young modulus of all bars in this and all subsequent examples is set to one. When solving the problem of maximizing the minimum eigenvalue (S) with $\bar{V}=1.2$ and $\bar{\gamma}=1$, we obtain $\lambda^{*}=4.9691 \cdot 10^{-2}$ and an optimal design $x^{*}$ shown in Figure 7-right. The next Figure 8 shows the influence of the optimal design on $\bar{V}$; we solve the same problem but with different bounds on the available volume, $\bar{V}=1.5$ and $\bar{V}=2.0$. The corresponding optimal eigenvalues are $\lambda^{*}=6.9899 \cdot 10^{-2}$ and $\lambda^{*}=10.811 \cdot 10^{-2}$, respectively. In all three problems, PENBMI needed about 130 inner iterations to find the optimal solution. The solution time was below one second.

Example 15 This academic example illustrates the possible nonuniqueness of solution to the problem $(\mathrm{S})$. Consider a $2 \times 3$ ground-structure with boundary conditions and load $(1,0)$ as 

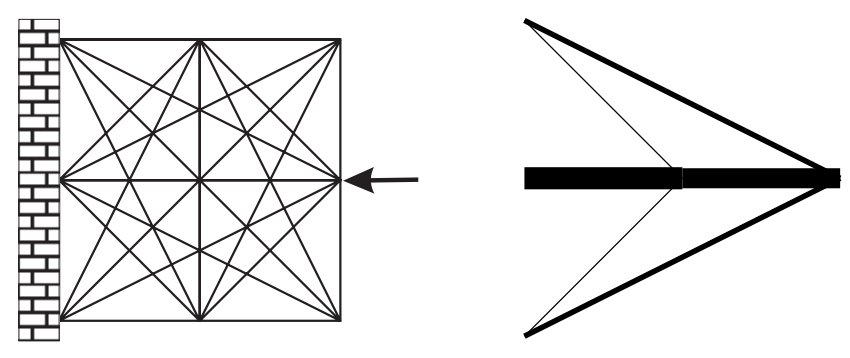

Figure 7: Three-by-three truss (Ex. 14): initial layout and optimal topology for $\bar{V}=1.2$
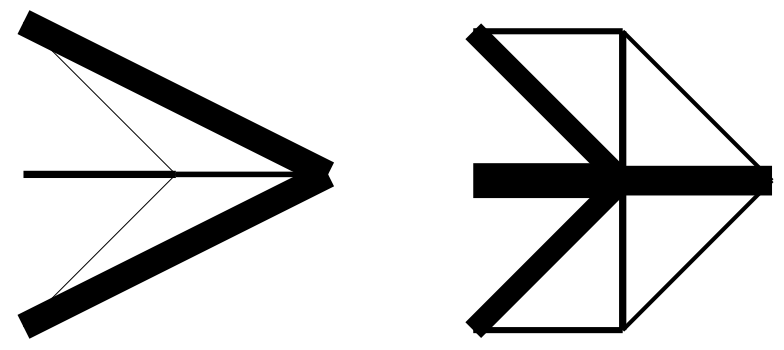

Figure 8: Three-by-three truss (Ex. 14): optimal topology for $\bar{V}=1.5$ and $\bar{V}=2.0$

depicted in Figure 9-left. Put $M_{0}=0, \bar{\gamma}=10$, and $\bar{V}=10$. The computed optimal structure $x^{*}$ is presented in Figure 9-right; the optimal objective function value of (S) is $\lambda^{*}=\lambda_{\min }\left(x^{*}\right)=$ 0.70711. While the volume constraint is active at $x^{*}$, the compliance constraint is inactive (more precisely, after calculating some $u^{*}$ corresponding to $x^{*}$, we have $\gamma^{*}:=f^{T} u^{*}=0.1<\bar{\gamma}=10$ ). Proposition 2.10 from [2] suggests that if we scale the solution $x^{*}$ by a certain factor $\mu$, we will still get a solution to problem (S). For instance, if we solve the same problem but with $\bar{V}=1.0$, then we will obtain a solution with the same $\lambda^{*}$ and with $\gamma^{*}=1.0$, i.e., still within the $\bar{\gamma}$ limits. Table 1 summarizes these numbers. It also presents the results for the case when $M_{0}=10$. In this case, the optimal solution is no longer scalable.

Table 1: Results of Example 15 for different data

\begin{tabular}{cccc}
\hline$M_{0}$ & $\bar{V}$ & $\gamma^{*}$ & $\lambda^{*}$ \\
\hline 0 & 1 & 1 & 0.70711 \\
0 & 10 & 0.1 & 0.70711 \\
\hline 10 & 1 & 1 & 0.08761 \\
10 & 10 & 0.1 & 0.41421 \\
\hline
\end{tabular}

Example 16 Here we demonstrate the possible nonuniqueness of solutions to the minimum volume problem (10). The purpose is to show that problems (10) and (S) are indeed not equivalent and one cannot assume to get a solution of the (nonlinear) problem (S) be solving the (linear) SDP counterpart to problem (10).

Consider the same ground-structure and boundary conditions as in Ex. 14 (see Fig. 7-left). The load vector, however, has changed to a single vertical force $(0,1)$ applied at the bottom-right 

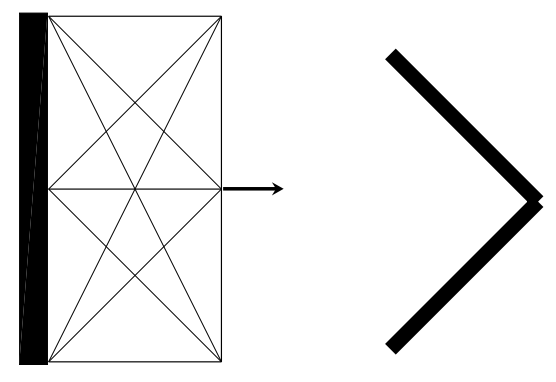

Figure 9: Example demonstrating possible nonuniqueness of solution of the (S) problem

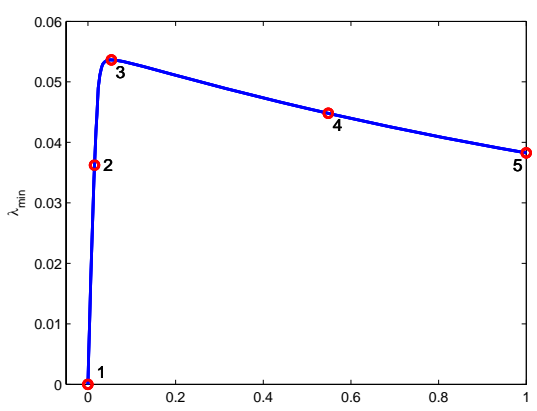

Figure 10: Example 16-graph of $\lambda_{\min }$ on interval between two structures of the same volume and compliance

node. Let further $\bar{\gamma}:=0.5$ and consider the single load min-volume problem without vibration constraint

$$
\begin{aligned}
& \min _{x \in \mathbb{R}^{m}, u \in \mathbb{R}^{n}} \sum_{i=1}^{m} x_{i} \\
& \text { subject to } \\
& \quad K(x) u=f, \\
& \quad f^{T} u \leq \bar{\gamma}, \quad i=1, \ldots, m . \\
& \quad x_{i} \geq 0, \quad
\end{aligned}
$$

This problem can be formulated as a linear program [1], and thus the set

$$
\mathcal{X}_{(18)}^{*}=\left\{x^{*} \mid \exists u^{*}:\left(x^{*}, u^{*}\right) \text { solves }(18)\right\}
$$

of solution structures of (18) is given by the set of all convex combinations of the most-left and most-right structure in Figure 11, i.e., by the set

$$
\mathcal{X}_{(18)}^{*}=\left\{(1-\mu) x^{1 *}+\mu x^{2 *} \mid \mu \in[0,1]\right\}
$$

where $x^{1 *}$ denotes the most-left and $x^{2 *}$ the most-right structure in Fig. 11. We have $\sum x_{i}^{*}=18$ and $f^{T} u^{*}=1$ for all $x^{*} \in \mathcal{X}_{(18)}^{*}$ and corresponding optimal displacement vectors $u^{*}$. Figure 10 shows the dependence of the minimum vibration eigenvalue on the parameter $\mu$ of this convex 

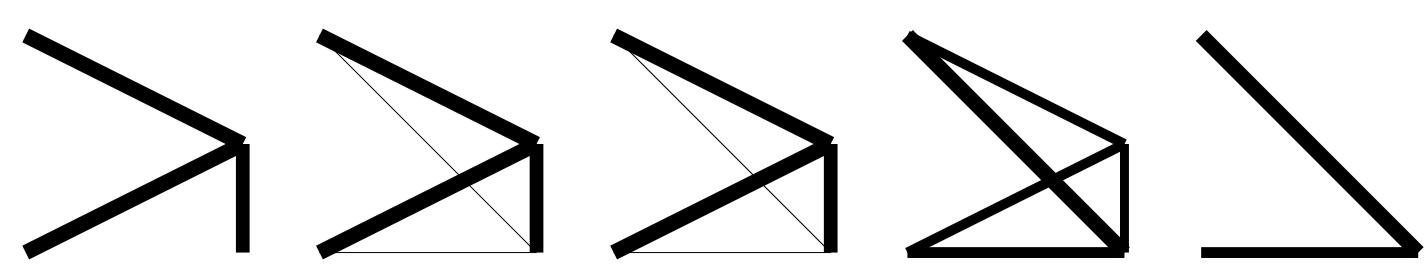

Figure 11: Example 16-structures corresponding to points 1-5 on the graph in Figure 10

combination, i.e., a plot of the function

$$
\mu \mapsto \lambda_{\min }\left((1-\mu) x^{1 *}+\mu x^{2 *}\right), \quad \mu \in[0,1] .
$$

The points $1-5$ in the plot correspond to the structures in Figure 11, left to right. We observe that $\lambda_{\min }$ is maximized at $\mu \approx 0.0536$, i.e., at structure number 3 . Let us now add the vibration constraint to problem (18); thus we arrive at problem (10). For example, put $\bar{\lambda}:=0.037$ which is the value of $\lambda_{\min }$ for structure number 2 in Figure 11. Then it is clear from the plot in Fig. 10 that any structure between truss number 2 and number 5 is a solution to problem (10), and the vibration constraint will be inactive for the structures strictly in between.

Example 17 We now present an example with multiple loads. Consider a $7 \times 3$ nodal grid with the ground-structure, boundary conditions and loads as depicted in Figure 12(a). Each of the forces $f_{1}=(-1,0)$ and $f_{2}=(0,1)$ represent an independent load case. The result of the standard minimum volume multiple-load problem (no vibration constraints) with $\bar{\gamma}=0.01$ is shown in Figure 12(b). When we consider both forces as a single load and solve a problem with no vibration constraints, we would obviously get a result consisting of a single rod between the two oposite forces. The volume of this structure is $V^{*}=35.485$. Figure 12(c) shows the result of the multiple load minimum eigenvalue problem (S) with bounds $\bar{\gamma}=0.01$ and $\bar{V}=40.0$. The optimal smallest eigenvalue is $\lambda^{*}=6.2216 \cdot 10^{-3}$. For a comparison, we also show a result of the single load problem (both forces considered as a single load) with $\bar{\gamma}=0.02$ and $\bar{V}=40.0$; the optimal structure with $\lambda^{*}=8.1674 \cdot 10^{-3}$ is presented in Figure 12(d). All solutions were obtained by PENBMI in less than 10 seconds.

Example 18 We consider the same problem scenario as in Example 14 but with a $7 x 7$ full ground-structure with 1176 potential bars; see Figure 13-left. In addition, we assign nonstructural mass of size 10 at the loaded node, i.e., $M_{0} \neq 0$. We solve the problem (S) with $\bar{\gamma}=1$ and $\bar{V}=3.0$. Figure 13-right shows the calculated optimal design $x^{*}$. The optimal eigenvalue is $\lambda^{*}=4.2383 \cdot 10^{-2}$. To solve the nonlinear SDP problem by PENBMI, we needed 143 inner iterations and $10 \mathrm{~min} 5 \mathrm{sec}$ of CPU time. Note that the optimization problem had 1177 variables, two matrix constraints of sizes $85 \times 85$ and $84 \times 84$ (one linear and one bilinear), one linear inequality constraint and bounds on all variables.

Example 19 Finally, we present a result of a three-dimensional example. The initial configuration is indicated in Figure 14-left: a ground-structure of $5 \times 3 \times 3$ nodes, each of them connected by a potential bar, resulting in 990 potential bars. All nodes on the left-hand side are fixed and the vertical force $(-1,0,0)$ is applied at the central right-hand side node. There is also a nonstructural mass of size 50 assigned to this node. We solve the problem (S) with $\bar{\gamma}=1$ and 
(a)

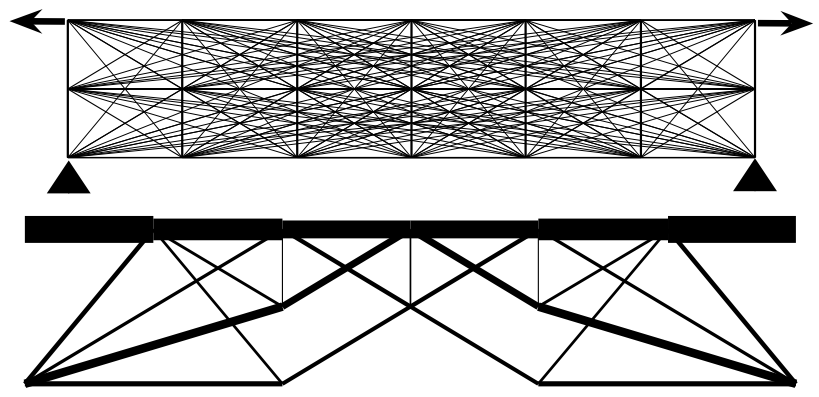

(b)

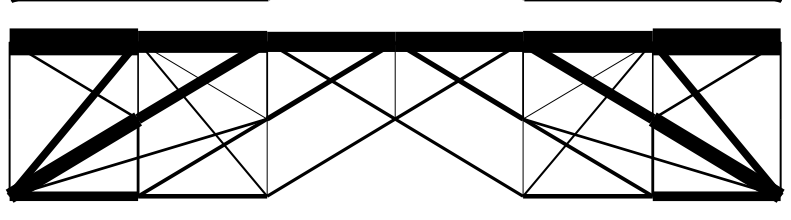

(d)

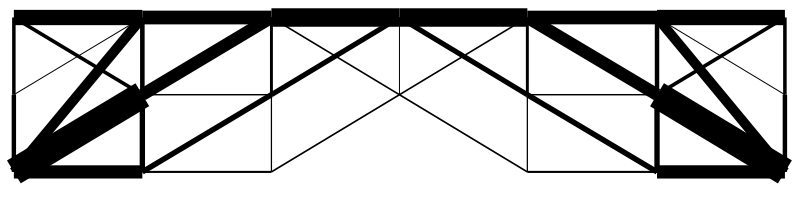

Figure 12: A medium size multiple-load example (Ex. 17): initial layout (a); optimal topology without (b) and with (c) vibration constraints; single-load optimal result with vibration constraints (d)

$\bar{V}=1.0$. Figure 14 shows the optimal structure from different viewpoints. The optimal value of the smallest eigenvalue is $2.4460 \cdot 10^{-4}$ Note that, at the optimum, the value of the compliance is $\gamma=0.184$, so the compliance constraint is inactive. When we decrease the bound on compliance to $\bar{\gamma}=0.05$, we get the simple design shown in Figure 15; this time the optimal eigenvalue is $2.1753 \cdot 10^{-4}$.

The optimization problems were again solved by PENBMI; to get a solution, it needed about 200 inner iterations and $10 \mathrm{~min}$ of CPU time.

\section{An extension: the multiple-mass problem}

Here we propose an extension of the original problem formulation (P) and its SDP reformulation (S), respectively. Assume that we have $N$ matrices $M_{0}^{(k)}, k=1, \ldots, N$, corresponding to $N$ different nonstructural masses that can be applied independently. For each mass we obtain a different minimal well-defined eigenvalue which is denoted by

$$
\left.\begin{array}{ll}
\lambda_{\min }\left(x, M_{0}^{(k)}\right)=\min \left\{\lambda \mid \exists w \in \mathbb{R}^{n}:\right. & K(x) w=\lambda\left(M(x)+M_{0}\right) w, \\
& w \notin \operatorname{ker}\left(M(x)+M_{0}^{(k)}\right)
\end{array}\right\} .
$$

Here we simply distinguish with respect to the particularly considered non-structural mass; compare to Notation 2 in Section 2.

Then the objective function $\lambda_{\min }(\cdot)$ in problem $(\mathrm{P})$ may be generalized to the worst-case minimal eigenvalue, i.e., to the function

$$
x \mapsto \min _{1 \leq k \leq N} \lambda_{\min }\left(x, M_{0}^{(k)}\right)
$$



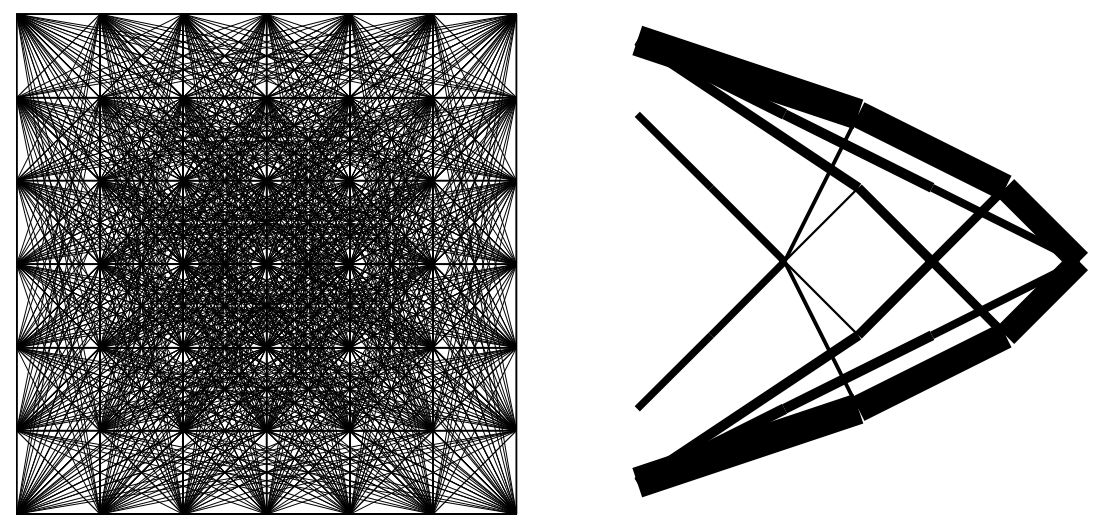

Figure 13: Example 18-a medium-size problem, initial layout and optimal topology

which is to be maximized. Problem $(\mathrm{P})$ becomes

$$
\begin{array}{ll}
\max _{x \in \mathbb{R}^{m}, u \in \mathbb{R}^{L \cdot n}} \min _{1 \leq k \leq N} \lambda_{\min }\left(x, M_{0}^{(k)}\right) & \\
\text { subject to } & \\
\qquad\left(\sum_{i=1}^{m} x_{i} K_{i}\right) u_{\ell}=f_{\ell}, & \ell=1, \ldots, L \\
f_{\ell}^{T} u_{\ell} \leq \bar{\gamma}, & \\
\sum_{i=1}^{m} x_{i} \leq \bar{V} & i=1, \ldots, L \\
x_{i} \geq 0, &
\end{array}
$$

By the same steps as above we transform (P) into an equivalent SDP and arrive at the following generalization of (S), for which theorems analogous to Thm. 9 and Thm. 10 hold.

$$
\begin{aligned}
& \max _{x \in \mathbb{R}^{m}, \lambda \in \mathbb{R}} \lambda \\
& \text { subject to } \\
& \begin{array}{ll}
\left(\begin{array}{cc}
\gamma & f_{\ell}^{T} \\
f_{\ell} & K(x)
\end{array}\right) \succeq 0, & \ell=1, \ldots, L \\
\sum_{i=1}^{m} x_{i} \leq \bar{V} & \\
x_{i} \geq 0, & i=1, \ldots, m \\
K(x)-\lambda\left(M(x)+M_{0}^{(k)}\right) \succeq 0, & k=1, \ldots, N .
\end{array}
\end{aligned}
$$

Because the mathematical structure of this formulation is the same as that of problem (S), we may use again the code PENBMI to solve this problem numerically; we may also construct an algorithm analogous to Algorithm 12. 

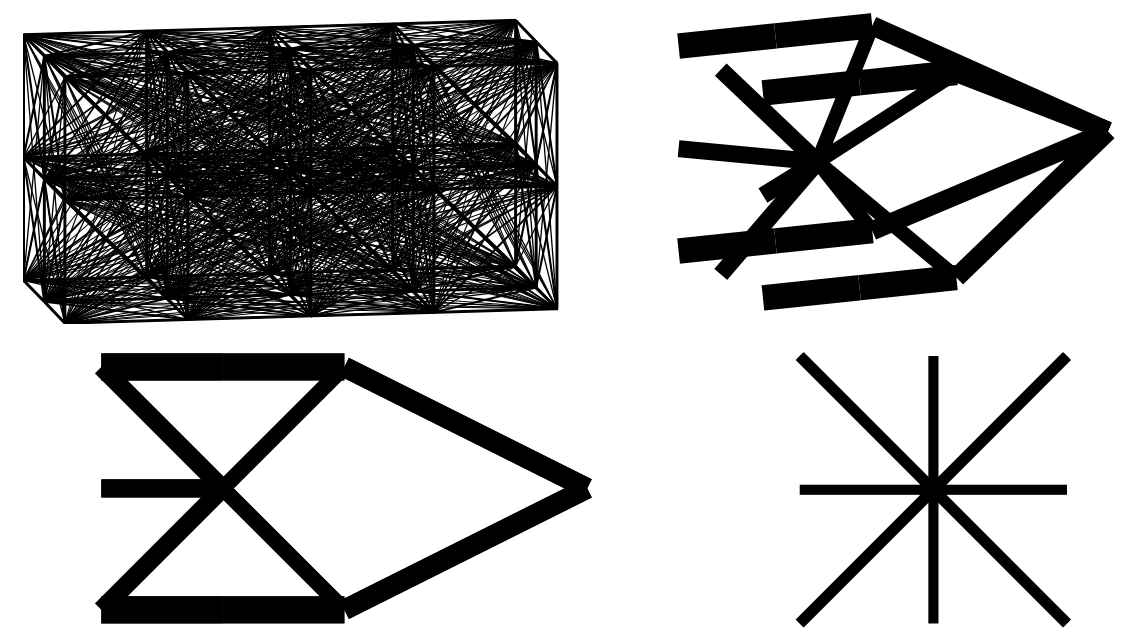

Figure 14: Example 19-a medium-size 3d problem, initial layout and optimal topology from different angles of view.
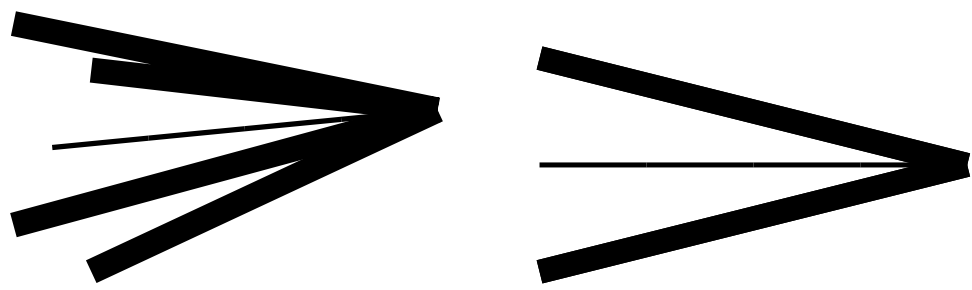

Figure 15: Example 19—a medium-size 3d problem, optimal topology for $\bar{\gamma}=0.05$.

Example 20 Consider a 3-by-3 truss with all nodes connected by potential bars. The nodes on the left-hand side are fixed in both directions, two nonstructural masses are placed in the corners on the right-hand side; see Figure 16-left. An external load is not applied, i.e., $L=0$. Figure 16-middle shows the optimal design for formulation $(\mathrm{S})$ when both masses are considered a "single" nonstructural mass. Figure 16-right presents the result of the multiple-mass formulation (20), where the two nonstructural masses are considered being independent from each other. The volume bound in both problems was $\bar{V}:=1$ and the resulting optimal eigenvalues were $\lambda^{*}=4.758 \cdot 10^{-3}$ in the single-mass case and $\lambda^{*}=7.365 \cdot 10^{-3}$ in the multiple-mass case. 

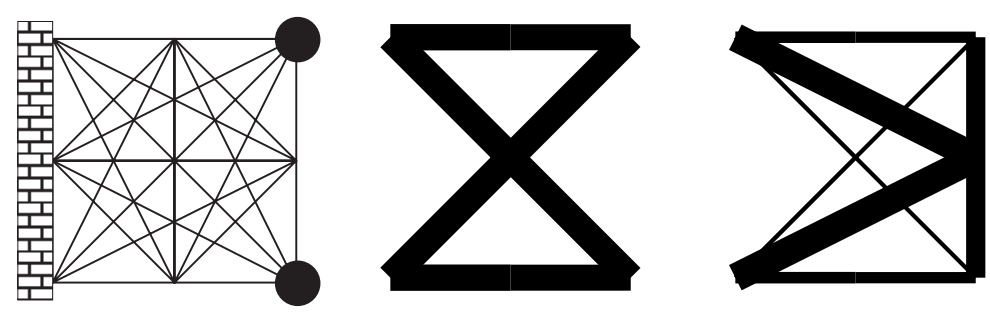

Figure 16: A multiple-mass problem (Ex. 20: initial layout (left), a "single-mass" result (middle) and a multiple-mass optimal structure (right)

\section{Acknowledgements}

The work was partially done while WA was visiting the Department of Mathematics, Technical University of Denmark, Kgs. Lyngby, Denmark, and MK was visiting the Institute for Mathematical Sciences, National University of Singapore, in 2006. The support and hospitality of these institutions is gratefully acknowledged.

This research was supported by the German-Czech DAAD-AV ČR project D-CZ 7/05-06 and by the Academy of Sciences of the Czech Republic through grant No. A1075402. 


\section{References}

[1] W. Achtziger, A. Ben-Tal, M. Bendsøe, and J. Zowe. Equivalent displacement based formulations for maximum strength truss topology design. IMPACT of Computing in Science and Engineering, 4:315-345, 1992.

[2] W. Achtziger and M. Kočvara. Structural topology optimization with eigenvalues. Technical Report No. 315, Institute of Applied Mathematics, University of Dortmund, Germany, February 2006.

[3] A. Ben-Tal and A. Nemirovski. Lectures on Modern Convex Optimization. MPS-SIAM Series on Optimization. SIAM Philadelphia, 2001.

[4] M. Bendsøe and O. Sigmund. Topology Optimization. Theory, Methods and Applications. Springer-Verlag, Heidelberg, 2002.

[5] R. Bhatia. Matrix Analysis. Springer Verlag, New York, 1996.

[6] R. Bhatia and R.-C. Li. On perturbations of matrix pencils with real spectra. II. Mathematics of Computation, 65:637-645, 1996.

[7] F. H. Clarke. Optimization and Nonsmooth Analysis. Wiley, New York, 1983.

[8] E. de Klerk. Aspects of Semidefinite Programming. Kluwer, 2002.

[9] F. R. Gantmacher. The Theory of Matrices, volume 1. Chelsea, New York, 1959.

[10] M. Kočvara, F. Leibfritz, M. Stingl, and D. Henrion. A nonlinear SDP algorithm for static output feedback problems in COMPlib. LAAS-CNRS research report no. 04508, LAAS, Toulouse, 2004.

[11] M. Kočvara and M. Stingl. PENNON-a code for convex nonlinear and semidefinite programming. Optimization Methods and Software, 18(3):317-333, 2003.

[12] M. Kočvara and M. Stingl. PENBMI User's Guide. Version 2.1., February 2006. http://www. penopt.com/.

[13] A. S. Lewis and M. L. Overton. Eigenvalue optimization. Acta Numerica, 5:149-190, 1996.

[14] J. Löfberg. YALMIP : A toolbox for modeling and optimization in MATLAB. In Proceedings of the CACSD Conference, Taipei, Taiwan, 2004. Available from http://control.ee.ethz.ch/ joloef/yalmip.php.

[15] D. Luenberger. Optimization by vector space methods. J. Wiley and Sons, 1997.

[16] M. Ohsaki, K. Fujisawa, N. Katoh, and Y. Kanno. Semi-definite programming for topology optimization of trusses under multiple eigenvalue constraints. Comp. Meth. Appl. Mech. Engng., 180:203-217, 1999.

[17] N. Olhoff. Optimal design with respect to structural eigenvalues. In F.P.J. Rimrott and B. Tabarott, editors, Theoretical and Applied Mechanics, Proc. XVth Int. IUTAM Congress, pages 133-149. North-Holland, 1980. 
[18] N. Olhoff and S. H. Rasmussen. On single and bimodal optimum bukling loads of clamped columns. Int. J. Solids Struct., 13:605-614, 1977.

[19] A. P. Seyranian and A. A. Mailybaev. Multiparameter stability theory with mechanical applications. World Scientific, Singapore, 2003.

[20] G. W. Stewart. Perturbation bounds for the definite generalized eigenvalue problem. Linear Algebra Appl., 23:69-86, 1979.

[21] M. Stingl. On the Solution of Nonlinear Semidefinite Programs by Augmented Lagrangian Methods. PhD thesis, Inst. of Appl. Math., University of Erlangen, 2005.

[22] T. Zhang, K. H. Law, and G. H. Golub. On the homotopy method for perturbed symmetric generalized eigenvalue problems. SIAM J. Sci. Comput., 19:1625-1645, 1998. 